\title{
Review
}

\section{DCBs as an adjuvant tool to DES for very complex coronary lesions}

\author{
Rima Chaddad ${ }^{1}$, Rami El-Mokdad ${ }^{2}$, Leontin Lazar ${ }^{3}$, Bernardo Cortese ${ }^{3,4, *}$ \\ ${ }^{1}$ Cardiology Department, Lebanese University, 00961 Beirut, Lebanon \\ ${ }^{2}$ Cardiology Department, Al-Zahraa University Hospital, 00961 Beirut, Lebanon \\ ${ }^{3}$ Clinica Polispecialistica San Carlo, Paderno Dugnano, 20037 Milano, Italy \\ ${ }^{4}$ Fondazione Ricerca e Innovazione Cardiovascolare, 20121 Milano, Italy \\ *Correspondence: bcortese@gmail.com (Bernardo Cortese) \\ Academic Editor: Jerome L. Fleg \\ Submitted: 20 October 2021 Revised: 30 November 2021 Accepted: 1 December 2021 Published: 14 January 2022
}

\begin{abstract}
Drug eluting stents (DES) have revolutionised interventional cardiology and currently represent the standard for percutaneous coronary interventions (PCI). However, due to several limitations, new strategies are required, especially in very complex lesions. Drug-coated balloons (DCB) offer an attractive therapeutic alternative, and have already obtained a Class I recommendation for the treatment of in stent restenosis (ISR) with Level A of evidence. Moreover, the role of DCB has been tested in several other settings, such as de novo large vessel disease, multivessel disease or very complex lesions, with promising results regarding safety and effectiveness. In this context, a hybrid strategy consisting in the use of a DES and DCB with the aim of reducing the amount of metal implanted and minimising the risk of ISR and stent thrombosis could become the solution for very complex lesions. Several important studies already demonstrated very good angiographic results in terms of late lumen loss and restenosis for this approach in bifurcation lesions. Moreover, for long diffuse coronary disease similar rates of MACE, TVR, and TLR at 2-year follow-up in comparison to a DES-alone strategy were found. What is more, the use of this strategy in high-bleeding risk patients could safely permit the practitioners to reduce the DAPT duration, as data is suggesting. As our daily practice already strongly incorporated this strategy and with more data expected from important trials, it is our strong believe that the hybrid approach can become a standard treatment choice in the near future.
\end{abstract}

Keywords: DCB; DES; Complex coronary lesions

\section{Introduction}

Balloon angioplasty or plain old balloon angioplasty (POBA) revolutionized interventional cardiology, as it offered an alternative to surgical treatment for coronary artery disease (CAD) [1]. However, elastic recoil and flowlimiting dissections and restenosis caused by cellular proliferation are the major drawbacks of this strategy [2]. By tackling dissections and eliminating elastic recoil, the use of intracoronary bare metal stent became the standard approach for percutaneous coronary interventions (PCI), but this strategy was limited by stent thrombosis and increased neointimal hyperplasia, leading to in-stent restenosis [3].

Drug-eluting stents (DES), which elute an antiproliferative drug (e.g., paclitaxel, sirolimus or analogues) to the vessel wall and reduce the in-stent restenosis rate, is now the cornerstone management during percutaneous coronary intervention [4]. However, there are still multiple lesions which PCI may not adequately solve, such as bifurcation, long, calcified, ostial lesions or chronic total occlusions (CTO). Moreover, late stent thrombosis and recurring events, dependency on prolonged dual antiplatelet therapy and restenosis led to a quest for new treatment modalities.

In this context, drug-coated balloons (DCB) offer an attractive therapeutic alternative, which consists in a combination of classical balloon angioplasty alongside with local drug delivery [5]. Several randomized trials have al- ready established the role of DCB in the treatment of instent restenosis of both DES and bare metal stents, thus obtaining a Class I recommendation for the treatment of ISR with Level A of evidence [6]. On the other hand, the use of DCB in de novo coronary artery disease, even though was demonstrated safe and effective in several clinical settings, is still to be acknowledged by international guidelines [7].

The purpose of this review is to outline different approaches and trial results with the use of DCB in combination with DES for de novo lesions and propose a novel design for future trials treating very complex coronary lesions.

\section{DCB design and procedural aspects}

A DCB is a non-stent based technology in which the effective homogenous delivery of anti-proliferative drugs is processed by the vessel wall through an inflated balloon. Both the polymer and stent platform are absent and the need for any foreign object within the vessel wall that might predispose to thrombus formation is completely eliminated. The drug coated balloon technology relies on the concept of targeted drug delivery, which helps in the rapid healing of the vessel wall and prevents the proliferation of smooth muscle cells.

Paclitaxel eluting balloons (PCB) are the first DCBs used in clinical practice and since their introduction, sev- 
eral new DCBs have been developed and are currently marketed in Europe. These devices vary in terms of the used materials for designing the balloon, the coating techniques, the choice of anti-proliferative drug and the release pattern of the drug at the site. As the most common dose used in the DCB design is $3 \mu \mathrm{g}$ paclitaxel $/ \mathrm{mm}^{2}$, with only few DCBs having different doses (Elutax SV/III-2.2 $\mu \mathrm{g}$ paclitaxel $/ \mathrm{mm}^{2}$, Danubio- $2.5 \mu \mathrm{g}$ paclitaxel $/ \mathrm{mm}^{2}$ and Agent-2 $\mu \mathrm{g}$ paclitaxel $/ \mathrm{mm}^{2}$ ) and no data to directly compare these devices to the standard dose DCBs in terms of efficacy, we cannot formulate any conclusions regarding the dose-dependent efficacy of Paclitaxel Eluting Balloons. While several studies have demonstrated the superiority of sirolimus-eluting stents over paclitaxel-eluting stents in terms of target-lesion revascularization (TLR), target-vessel revascularization (TVR), re-stenosis rate and major adverse cardiac event (MACE) [8], these results have not been yet described for DCB, as two important studies only hypothesized and managed to demonstrate the noninferiority of the new Sirolimus DCB $[9,10]$.

Regarding the technical aspects of the use of this device, a good lesion preparation by a $0.8-1: 1$ ratio predilatation using a noncompliant balloon is mandatory for most of the uncomplicated lesions. Furthermore, for more complex lesions, plaque modification balloons or debulking devices may be used. After a good preparation result (defined as a good balloon expansion, less than $30 \%$ of residual stenosis, a TIMI flow grade 3 and the absence of flow-limiting dissection) is obtained, DCB can be used, and a bailout approach with a stent implantation can be performed in the presence of an unsatisfactory angiographic result, mainly flow-limiting dissections [11,12].

Moreover, in order to better evaluate the postpredilatation result and to improve the outcomes of DCB use, several studies have investigated the role of intravascular imaging in this setting. In a prospective study including patients with de novo lesions treated with Paclitaxel DCB, Ann SH et al. [13] performed serial angiographic, optical coherence tomography and fractional flow reserve measurements before, after and at 9 months follow-up. They observed that $66.6 \%$ of the dissections initially seen after lesion preparation were sealed on follow-up optical coherence tomography (OCT) [13]. Moreover, the fractional flow reserve (FFR) distal to the target lesion significantly increased after DCB, with preserved results at follow-up, thus demonstrating a high percentage of healing for the residual dissections, which were a main reason of concern for DCB use [13]. In another study, Poerner TC et al. [14] used a protocol for a non-stent based approach, in which after predilatation, if the residual stenosis was $<40 \%$, with FFR $>0.8$ and no severe dissection, the target lesion was treated using the DCB. At follow-up OCT, the lumen diameter showed a trend toward progressive increase, thus the strategy was feasible and safe in stable CAD [14]. Furthermore, intravascular ultrasound (IVUS) was also used in dif- ferent studies, and the findings led to a better understanding of the DCB use. Using IVUS at the index procedure and at 9 months follow-up in patients treated with DCB, Yamamoto et al. [15] were able to observe a significantly increased lumen volume, with mean plaque volume significantly decreased at 9 months, while $74.1 \%$ of the analysed lesions exhibited late lumen enlargement. In another study, Sato A et al. [16] used a dedicated protocol for treating coronary lesions, using directional coronary atherectomy, followed by DCB under IVUS guidance, with follow-up angiography and IVUS performed at 6-10 months. They found no TLRs or TVRs after this period, despite a rate of $13 \%$ minor dissections showed by the index procedure IVUS. Intravascular imaging also described a significantly reduction of diameter stenosis and plaque area, thus, this strategy appeared to be safe and efficient and once more demonstrated the importance of intravascular imaging when using a DCB, as minor dissections, properly evaluated this way, can remain stent-free, having a high-rate of healing [16].

\section{Clinical use}

A tremendous work regarding the use of DCB has been carried on in the last decade and, as a result, there are currently multiple studies that demonstrated the safety and effectiveness of this approach in multiple settings, especially in complex and high-risk indicated procedures (CHIP) patients.

Firstly, regarding small vessels disease, in the PICCOLETO trial, the DCB approach was superior to DES in terms of angiographic late lumen loss (LLL) [17], with similar results found in the randomised controlled multicentre BELLO study (Balloon Elution and Late Loss Optimization), which also found similar rates of restenosis and revascularization when compared to a DES [18]. Moreover, while RESTORE and BASKET SMALL 2 trials demonstrated the non-inferiority of a DCB vs DES regarding insegment diameter stenosis at 9 month follow-up, respectively clinical efficacy at 12 month follow-up, the PICCOLETO 2 trial found DCB to be superior to DES in terms of LLL and comparable in terms of clinical outcomes [19].

As the high efficacy of a non-stent based approach in this setting was, therefore, demonstrated, the real question which is still in need for a well-documented answer is if a DCB could also perform with the same results in larger vessels and, what is more, in complex lesions. For this reason, the FALCON registry enrolled 757 patients with large coronary artery disease treated with DCB and showed a good safety and efficacy profile at 1-year follow-up [20]. In another study which enrolled patients with de novo coronary lesions in large vessels, who also had a high bleeding risk, DCB was found superior to BMS in terms of MACE at 9 months and with no acute vessel closure described. This study is not only suggesting once more that DCB may be feasible and safe in large coronary arteries as well, but also emphasis another major clinical implication of the stent- 


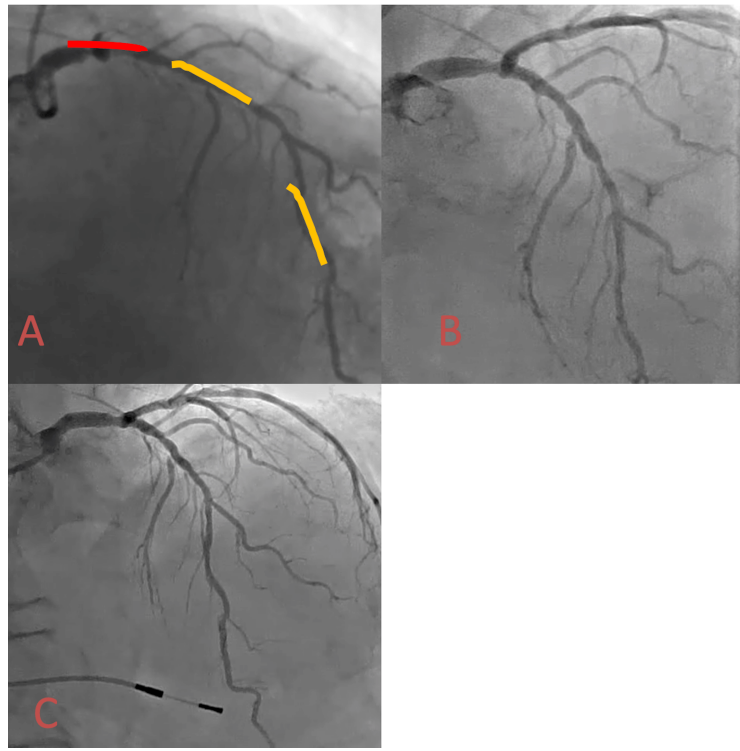

Fig. 1. Hybrid strategy for treating a critical LM-LAD long diffuse disease. (A) Basal angiography showing the distal LM critical stenosis and a long diffuse LAD disease. The planned strategy was to use a DES only for the LM-proximal LAD (red line), followed by 2 long DCB for the mid and distal LAD (yellow lines). (B) Final angiographic result showing no important dissection, with optimal distal flow. (C) 6 months follow-up angiography showing the vessel healing.

free approach: the possibility of a safer PCI for the highbleeding patients [21]. As a precise indication regarding the dual-antiplatelet (DAPT) duration after DCB use is still lacking, the shortest DAPT duration (even $<1$ month) in high bleeding risk patients is recommended by most of the experts [11,21]. Moreover, even a single antiplatelet regimen, which was adopted in $17 \%$ of the only-DCB treated patients in the DCB RISE registry proved to be safe for these high-bleeding risk patients, as no thrombotic events were reported after 12 months of follow-up [22]. All of these findings are not only suggesting the benefits of the DCB use in frail patients, but are also highlighting the urgent need for data, in order to be able to reduce the DAPT duration.

With growing evidence in favour of the use of DCB in de novo vessels, regardless of the size, the EASTBOURNE registry addressed real world complex patients suggesting that this approach could be suitable in multivessel disease [23] and while data is still scarce regarding other complex settings such as bifurcations, CTO or acute coronary lesions, the available results from multiple studies are promising. For example, ST-elevation myocardial infarction patients have been enrolled and randomised to either DCB or DES strategy in the REVELATION trial. At 9 months angiographic follow-up, there were no significant differences between the DCB and DES groups regarding fractional flow reserve [24]. Moreover, at 2 year follow-up, there were comparable clinical outcomes between the 2 groups, with only one additional event occurring in the DCB group between 9 and 24 months [25]. With data from ongoing trials such as PICCOLETO III (very long lesions, chronic total occlusions) and TRANSFORM II still waited to demonstrate the safety and effectiveness of DCB use in very complex lesions, it is now clear that DCBs are most likely to play a central role in the treatment of these patients as well, alone, or as part of a "hybrid strategy".

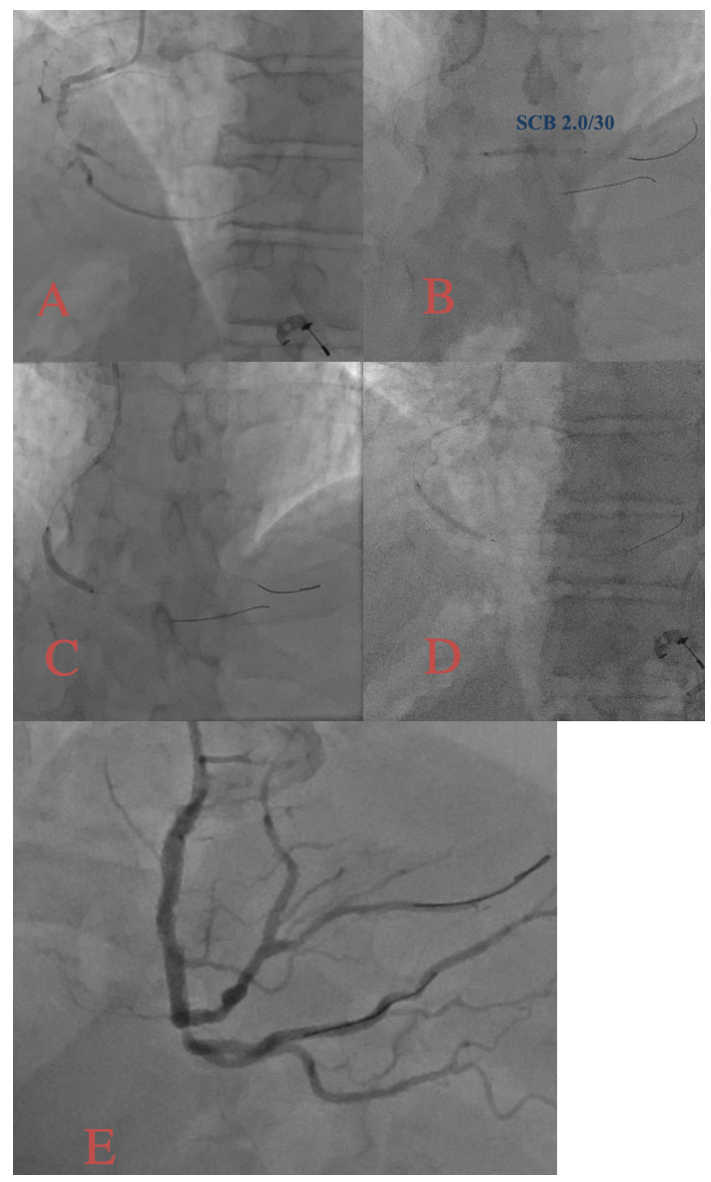

Fig. 2. Hybrid strategy for treating sub-occlusive small right coronary artery, with distal diffuse disease. (A) Basal angiography showing diffuse $\mathrm{RCD}$ disease with a sub-occlusive lesion in the mid segment. (B,C) Showing sirolimus DCB delivery. (D) Showing stent implantation. (E) Showing final result.

\section{Hybrid approach}

The main concept of a "hybrid strategy" is to reduce the amount of metal implanted in complex lesions, as it has been demonstrated that the stent length is an independent predictor for ISR and stent thrombosis, $>60 \mathrm{~mm}$ overlapping DES being associated with high TLR rates of approximatively $24 \%$. Moreover, the presence of long metal devices in the coronary arteries may affect the restoration of the segment's vasomotion, promote neoatherosclerosis and 


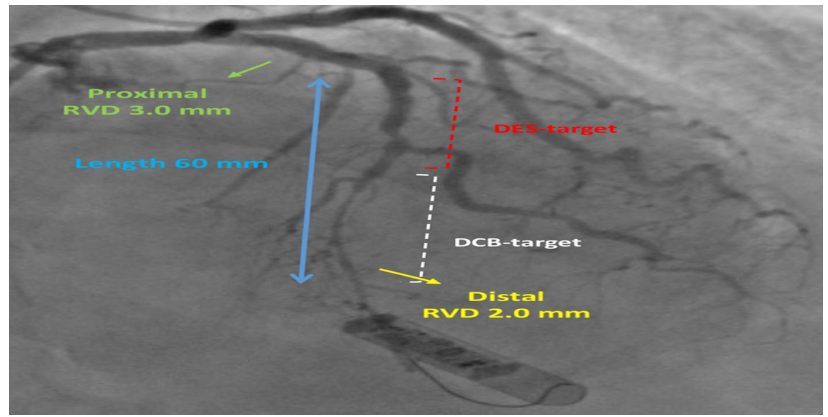

Fig. 3. Hybrid procedure DCB + DES in treating complex CAD. The Figure depicts a treatment strategy planning for a hybrid approach to a complex, long, diffuse LAD disease.

compromise potential future surgical options [26]. Thus, reducing the stent length by using DCBs, not only that is safe and effective, with demonstrated benefits on late lumen enlargement and non-inferiority in terms of LLL and stenosis diameter, but is also of great importance for the very long-term outcomes, as it preservers the potential future surgical by-pass option, in particular for mid/distal LAD.

The BIOLUX-I trial first studied this approach using an everolimus DES for the main branch and a paclitaxel DCB in the side branch for the treatment of bifurcation lesions, and the authors considered this approach to be safe and effective [27]. The DEBSIDE study also described very low LLL and low restenosis rates in the side branch ostium after applying a hybrid approach using a final inflation of a DCB in the side branch after placement of a DES in the main branch [28]. Using a similar approach, the BEYOND study also succeeded in showing good angiographic results at the 9-month follow-up in de novo non-LM bifurcations [29].

In another small study in patients with high restenosis risk, Basavarajaiah et al. [30] tested a strategy which implied using DCB alone in treating coronary lesions and in case of suboptimal result after predilatation to implant a DES in the same lesion. After a hybrid approach was applied in 46 patient (57 lesions), the results were encouraging [30], suggesting a synergic effect with dual drug elution and these results drove the authors into investigating a similar scenario in another larger study [31,32]. The use of a hybrid approach, both as a bailout or planned strategy was reported to reduce the stent length in long lesions, with similar rates of MACE, TVR, and TLR at 2-year follow-up in comparison to a DES-alone strategy [26].

As these results boosted the hope for an alternative for full metal jacket in very long diffuse lesions, thus reducing the very late risk of ISR and stent thrombosis, more data is expected to confirm the safety and efficacy of hybrid DES/DCB approach for the treatment of de-novo diffuse CAD from the HYPER pilot study (ClinicalTrials.gov Identifier: NCT03939468). In this study a hybrid strategy was defined as overlapping or slightly $(2-3 \mathrm{~mm})$ superim- posing a new generation DES implantation for a de novo lesion (located in the larger, more proximal part of the vessel) and DCB inflation for a concomitant de novo small vessel disease.

Using a similar aspect, Figs. 1,2,3 are relevant for our current practice in diffuse CAD and illustrate the good results obtained using a hybrid strategy for this challenging setting. What is more, this approach not only reduces the risk of in-stent restenosis and thrombosis, but could also represent a future standard strategy in high-bleeding risk patients, as it may allow the practitioners to reduce the duration of the DAPT regimen, as the DEBATE trial hypothesized (ClinicalTrials.gov Identifier: NCT04814212).

\section{Conclusions}

As de-novo diffuse CAD remains a challenge for interventional cardiologists and current data suggests that a DCB approach is safe and efficient in coronary de novo lesions, a hybrid approach using a DES/DCB treatment may improve the outcome of these complex patients. Moreover, this approach could reduce the DAPT regimen duration, thus offering a safer alternative comparing to DES for highbleeding risk patients. There are currently important large studies on-going which are expected to provide solid information regarding the performance of this strategy not only in diffuse $\mathrm{CAD}$, but also in bifurcation lesions or multi vessels disease, with promising preliminary results suggesting that this may become a standard treatment choice in the near future.

\section{Author contributions}

$\mathrm{RC}$ conceived and designed the analysis, collected the data, contributed data or analysis tools, wrote the paper. REM wrote the paper, contributed data or analysis tools. LL conceived and designed the analysis, collected the data, contributed data or analysis tolls, wrote the paper. BC conceived and designed the analysis, collected the data, contributed data or analysis tools, wrote the paper, feedback and update version of the paper.

\section{Ethics approval and consent to participate}

Not applicable.

\section{Acknowledgment}

Not applicable.

\section{Funding}

This research received no external funding.

\section{Conflict of interest}

The authors declare no conflict of interest. Bernardo Cortese is serving as one of the Guest editors of this journal. We declare that Bernardo Cortese had no involvement in the peer review of this article and has no access to infor- 
mation regarding its peer review. Full responsibility for the editorial process for this article was delegated to Jerome L. Fleg.

\section{References}

[1] Levine GN, Bates ER, Blankenship JC, Bailey SR, Bittl JA, Cercek B, et al. 2011 ACCF/AHA/SCAI Guideline for Percutaneous Coronary Intervention. A report of the American College of Cardiology Foundation/American Heart Association Task Force on Practice Guidelines and the Society for Cardiovascular Angiography and Interventions. Journal of the American College of Cardiology. 2011; 58: e44-122.

[2] Waksman R, Pakala R. Drug-eluting balloon: the comeback kid? Circulation Cardiovascular Interventions. 2009; 2: 352-358.

[3] Sigwart U, Puel J, Mirkovitch V, Joffre F, Kappenberger L. Intravascular Stents to Prevent Occlusion and re-Stenosis after Transluminal Angioplasty. New England Journal of Medicine. 1987; 316: 701-706.

[4] Morice M, Serruys PW, Sousa JE, Fajadet J, Ban Hayashi E, Perin M, et al. A Randomized Comparison of a SirolimusEluting Stent with a Standard Stent for Coronary Revascularization. New England Journal of Medicine. 2002; 346: 1773-1780.

[5] Rosenberg M, Waliszewski M, Chin K, Ahmad WAW, Caramanno G, Milazzo D, et al. Prospective, Large-Scale Multicenter Trial for the Use of Drug Coated Balloons (DCB) in Coronary Lesions: The DCB-Only All- Comers Registry. Catheterization and Cardiovascular Interventions. 2018; 93: 181-188.

[6] Neumann FJ, Sousa-Uva M, Ahlsson A, Alfonso F, Banning AP, Benedetto U, et al. ESC Scientific Document Group, 2018 ESC/EACTS Guidelines on myocardial revascularization. European Heart Journal. 2019; 40: 87-165.

[7] Waksman R, Serra A, Loh JP, Malik FT, Torguson R, Stahnke S, et al. Drug-coated balloons for de novo coronary lesions: results from the Valentines II trial. EuroIntervention. 2013; 9: 613-619.

[8] Zhang X, Xie J, Li G, Chen Q, Xu B. Head-to-head comparison of sirolimus-eluting stents versus paclitaxel-eluting stents in patients undergoing percutaneous coronary intervention: a metaanalysis of 76 studies. PLoS ONE. 2014; 9: e97934.

[9] Ali RM, Abdul Kader MASK, Wan Ahmad WA, Ong TK, Liew HB, Omar A, et al. Treatment of Coronary Drug-Eluting Stent Restenosis by a Sirolimus- or Paclitaxel-Coated Balloon. JACC: Cardiovascular Interventions. 2019; 12: 558-566.

[10] Cortese B, Caiazzo G, Di Palma G, De Rosa S. Comparison between Sirolimus- and Paclitaxel-Coated Balloon for Revascularization of Coronary Arteries: the SIRPAC (SIRolimusPAClitaxel) Study. Cardiovascular Revascularization Medicine. 2021; 28: 1-6.

[11] Cortese B, Berti S, Biondi-Zoccai G, Colombo A, Limbruno U, Bedogni F, et al. Drug-coated balloon treatment of coronary artery disease: a position paper of the Italian Society of Interventional Cardiology. Catheterization and Cardiovascular Interventions. 2014; 83: 427-435.

[12] Cortese B, Silva Orrego P, Agostoni P, Buccheri D, Piraino D, Andolina G, et al. Effect of Drug-Coated Balloons in Native Coronary Artery Disease Left with a Dissection. JACC: Cardiovascular Interventions. 2015; 8: 2003-2009.

[13] Ann SH, Her A, Singh GB, Okamura T, Koo B, Shin E. Serial Morphological and Functional Assessment of the Paclitaxelcoated Balloon for de Novo Lesions. Revista Espanola De Cardiologia. 2016; 69: 1026-1032.

[14] Poerner TC, Duderstadt C, Goebel B, Kretzschmar D, Figulla HR, Otto S. Fractional flow reserve-guided coronary angioplasty using paclitaxel-coated balloons without stent implantation: feasibility, safety and 6-month results by angiography and optical coherence tomography. Clinical Research in Cardiology. 2017;
106: 18-27.

[15] Yamamoto T, Sawada T, Uzu K, Takaya T, Kawai H, Yasaka Y. Possible mechanism of late lumen enlargement after treatment for de novo coronary lesions with drug-coated balloon. International Journal of Cardiology. 2020; 321: 30-37.

[16] Sato A, Kijima M, Ichimura S, Yaegashi D, Anzai F, Shimizu T, et al. Short-term outcome of percutaneous coronary intervention with directional coronary atherectomy followed by drug-coated balloon: a preliminary report. Cardiovascular Intervention and Therapeutics. 2019; 34: 149-154.

[17] Cortese B, Micheli A, Picchi A, Coppolaro A, Bandinelli L, Severi $\mathrm{S}$, et al. Paclitaxel-coated balloon versus drug-eluting stent during PCI of small coronary vessels, a prospective randomised clinical trial. the PICCOLETO study. Heart. 2010; 96: 12911296.

[18] Latib A, Colombo A, Castriota F, Micari A, Cremonesi A, De Felice F, et al. A Randomized Multicenter Study Comparing a Paclitaxel Drug-Eluting Balloon with a Paclitaxel-Eluting Stent in Small Coronary Vessels: the BELLO (Balloon Elution and Late Loss Optimization) study. Journal of the American College of Cardiology. 2012; 60: 2473-2480.

[19] Cortese B, Di Palma G, Guimaraes MG, Piraino D, Orrego PS, Buccheri $\mathrm{D}$, et al. Drug-Coated Balloon Versus Drug-Eluting Stent for Small Coronary Vessel Disease: PICCOLETO II Randomized Clinical Trial. JACC: Cardiovascular Interventions. 2020; 13: 2840-2849.

[20] Widder JD, Cortese B, Levesque S, Berliner D, Eccleshall S, Graf $\mathrm{K}$, et al. Coronary artery treatment with a urea-based paclitaxel-coated balloon: the European-wide FALCON allcomers DCB Registry (FALCON Registry). EuroIntervention. 2019; 15: e382-e388.

[21] Rissanen TT, Uskela S, Eränen J, Mäntylä P, Olli A, Romppanen $\mathrm{H}$, et al. Drug-coated balloon for treatment of de-novo coronary artery lesions in patients with high bleeding risk (DEBUT): a single-blind, randomised, non-inferiority trial. The Lancet. 2019; 394: 230-239.

[22] Cortese B, D'Ascenzo F, Fetiveau R, Balian V, Blengino S, Fineschi M, et al. Treatment of coronary artery disease with a new-generation drug-coated balloon: final results of the Italian Elutax SV rEgistry-DCB-RISE. Journal of Cardiovascular Medicine. 2018; 19: 247-252.

[23] Cortese B, Testa L, Di Palma G, Heang TM, Bossi I, Nuruddin AA, et al. Clinical performance of a novel sirolimus-coated balloon in coronary artery disease: EASTBOURNE registry. Journal of Cardiovascular Medicine. 2021; 22: 94-100.

[24] Vos NS, Fagel ND, Amoroso G, Herrman JR, Patterson MS, Piers LH, et al. Paclitaxel-Coated Balloon Angioplasty Versus Drug-Eluting Stent in Acute Myocardial Infarction: the REVELATION Randomized Trial. JACC: Cardiovascular Interventions. 2019; 12: 1691-1699.

[25] Niehe SR, Vos NS, Van Der Schaaf RJ, Amoroso G, Herrman JR, Patterson MS, et al. Two-Year Clinical Outcomes of the REVELATION Study: Sustained Safety and Feasibility of PaclitaxelCoated Balloon Angioplasty Versus Drug-Eluting Stent in Acute Myocardial Infarction. Journal of Invasive Cardiology. 2021. (in press)

[26] Costopoulos C, Latib A, Naganuma T, Sticchi A, Figini F, Basavarajaiah S, et al. The role of drug-eluting balloons alone or in combination with drug-eluting stents in the treatment of de novo diffuse coronary disease. JACC. Cardiovascular Interventions. 2013; 6: 1153-1159.

[27] Worthley S, Hendriks R, Worthley M, Whelan A, Walters DL, Whitbourn R, et al. Paclitaxel-eluting balloon and everolimuseluting stent for provisional stenting of coronary bifurcations: 12-month results of the multicenter BIOLUX-I study. Cardiovascular Revascularization Medicine. 2015; 16: 413-417. 
[28] Berland J, Lefèvre T, Brenot P, Fajadet J, Motreff P, Guerin P, et al. DANUBIO - a new drug-eluting balloon for the treatment of side branches in bifurcation lesions: six-month angiographic follow-up results of the DEBSIDE trial. EuroIntervention. 2015; 11: $868-876$

[29] Jing Q, Zhao X, Han Y, Gao L, Zheng Y, Li Z, et al. A drugeluting Balloon for the trEatment of coronarY bifurcation lesions in the side branch: a prospective multicenter ranDomized (beyond) clinical trial in China. Chinese Medical Journal. 2020; 133: 899-908.

[30] Basavarajaiah S, Latib A, Hasegawa T, Rezq A, Takagi K, Na- ganuma T, et al. Assessment of Efficacy and Safety of Combining "Paclitaxel" Eluting Balloon and "Limus" Eluting Stent in the same Lesion. Journal of Interventional Cardiology. 2013; 26: 259-263.

[31] Basavarajaiah S, Latib A, Shannon J, Naganuma T, Sticchi A, Bertoldi L, et al. Drug-Eluting Balloon in the Treatment of inStent Restenosis and Diffuse Coronary Artery Disease: RealWorld Experience from our Registry. Journal of Interventional Cardiology. 2014; 27: 348-355.

[32] Cortese B. Drug-Coated Balloons. Springer: Germany. 2019. 